\title{
Bund Schweizerischer Atemlehrer
}

Integrale Atemschulung - Lehrweise Wolf

\section{WER SIND WIR ?}

Wir sind über hundert Atemlehrer, die vielerorts in der Schweiz und in Deutschland tätig sind. Unsere berufsbegleitende Ausbildung erstreckt sich über mindestens zweieinhalb Jahre. Sie wird von der Atemschule WOLF mit Sitz in Brugg vermittelt.

\section{UNSER ZIEL}

Unsere Atem- und Körperschulung ist auf die Gesundheitsvorsorge, -erhaltung und -wiederherstellung ausgerichtet. Wir setzen dort an, wo der Mensch in Folge von Hetze, Leistungsdruck und Belastung jeglicher Art, sein psychisches Gleichgewicht verlieren kann, oder bereits verloren hat und zudem durch Bewegungsmangel mit den verschiedensten organischen Beschwerden konfrontiert wird. Durch Atemschulung und ökonomisches Einteilen seiner Kräfte lernt der gehetzte Mensch seine nervlich psychische Stabilität wieder aufzubauen. Sie lässt inn den Stress verkraften und befreit von möglichen Abhängigkeiten wie Rauchen, Alkohol und Essgier.

\section{Bund Schweizerischer Atemlehrer - Schule Wolf} Im Einsatz für sie

\section{UNSERE TAETI GKEIT}

In wöchent 1 ichen Uebungsstunden und einwöchigen Ferienkursen führen wir Interessenten aller Bevölkerungsschichten in die Technik des richtigen Atmens und in das bewusste Erleben des Atems ein. Mit gezielten Bewegungen in Verb indung mit rhythmischem Atmen verbessern und beeinflussen wir die Tätigkeit aller Organsysteme, damit diese ihre optimale Leistung erbringen können. Im Wechse1 von Bewegung und Ruhe lassen wir der Erholung und Erneuerung Raum.

Lernen Sie uns und unsere Arbeit kennen.

Die Atemlehrerin oder der Atemlehrer in Itrer Umgebung freut sich, Sie unverbindlich zu einer Lektion einzuladen.

Information: - zur weiteren Information steht Ihnen unsere Dokumentation mit der AnschriftenListe der Aktiv - Mitglieder des Atembundes zur Verfijgung.

- das Buch "Integrale Atemschulung" von Kiara Wolf, erschienen im Humata Verlag.

\section{Bund Schweizerischer Atemlehrer - Schule Wolf}

im Einsatz für sie

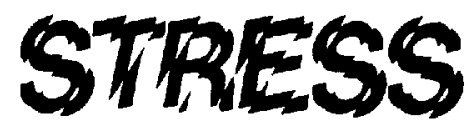

Jede Woche 1 Stunde sinnvolle Atem- und Körperschulung

O Nervlich - psychisches Gleichgewicht

$O$ Korizentration durch Optimieren der Atmung

durch Entspannungs- und Stabil isierungs tubungen - Eutonie durch Lun jenhygiene- und Organubungen
O verbesserte Sauerstoff - Sätigung

0 konzentrationsvermögen

O Nervlich - psychisches Gleichgew icht
O Gesunde Leistungssteigerung

Cund Schweiz. Atemlehrer UIrich Lang Reucrenettestr. 3 2502 Biel Tel.032/42 5759 durch Ausschöpfen der Erhoiungsphasen in Atmung und Bewegung durch rhythmisches Atmen durch vollen, aber ökonomischen Einsatz mit Rucksicht auf die Atem- und kreislaufkapazität
Reuch Lang

Reuchenet testr.
2502 Biel
rel.032/42 5759
Scheiz. Atemschule :OLLF

Wi1denra in
5200 Brugg

5200 Brugg 2296
Scheiz. Atemschule IriLF

ildenra in 20

5200 Brugg 2296 